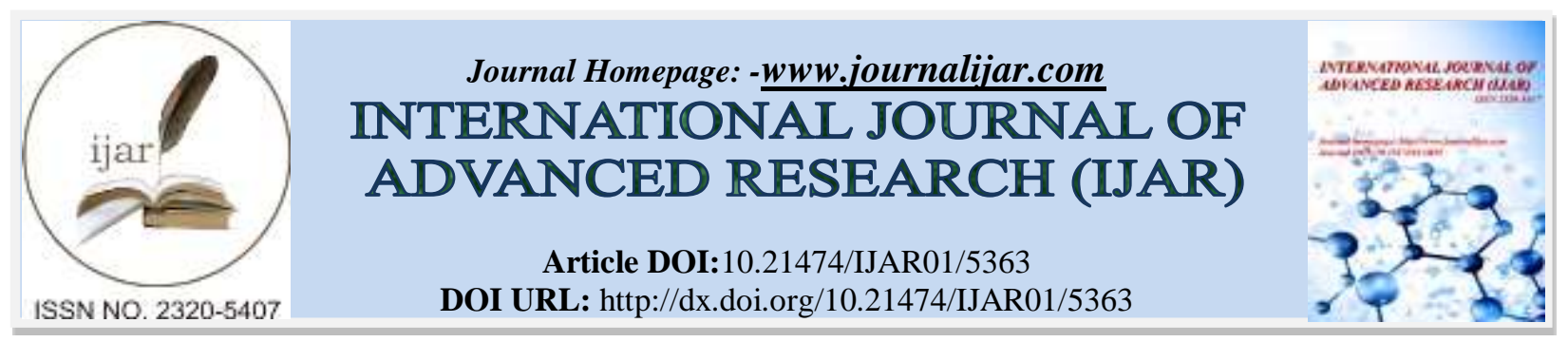

RESEARCH ARTICLE

\title{
A STUDY OF CEREBRAL VENOUS THROMBOSIS IN PREGNANCY AND PUERPERIUM.
}

Dr. Ph. Priyadarshini ${ }^{1}$, Dr. Munikrishna ${ }^{2}$, Dr. Anil Kumar Sakalecha ${ }^{3}$ and Dr. Gomathy. $\mathbf{E}^{4}$.

1. Post graduate student in the Department of Obstetrics and Gynecology, Sri Devraj URs Medical college, Sri Devraj URs Acadamy of higher Education and Research (SDUAHER), Kolar, Karnataka, India.

2. Professor in the Department of Obstetrics and Gynecology, Sri Devraj URs Medical college, Sri Devraj URs Acadamy of higher Education and Research (SDUAHER), Kolar, Karnataka, India.

3. Professor in the Department of Radiodignosis, Sri Devraj URs Medical college, Sri Devraj URs Acadamy of higher Education and Research (SDUAHER), Kolar, Karnataka, India.

4. Professor in the Department of Obstetrics and Gynecology, Sri Devraj URs Medical college, Sri Devraj URs Acadamy of higher Education and Research (SDUAHER), Kolar, Karnataka, India.

\section{Manuscript Info}

\section{Manuscript History}

Received: 08 July 2017

Final Accepted: 10 August 2017

Published: September 2017

\begin{abstract}
Background: We reported a case series of cerebral vein thrombosis (CVT) in women who are pregnant or in puerperium period. We evaluated the clinical features of pregnancy-related CVT populations, including disease onset and development, imaging features, diagnosis, treatments, and patient prognosis. Methods: This study was a retrospective case series of 6 patients with diagnosis of CVT, who were admitted and was treated in R.L. Jalappa Hospital and research center, Tamaka, Kolar, Karnataka. The duration of the study was one year from October 2015 to October 2016. Results: All the patients had no history of thrombosis before. They were treated conservatively with low molecular weight heparin and mannitol. They did not have other possible risk factors for CVT. Headache was the most common symptoms and seen in 6/6 patients (100\%) followed by vomiting and seizures.

Conclusion: We found that high rate of CVT in women with pregnancy and puerperium and indicates that it needs be considered as a specific risk factor and should also be considered by healthcare system in order to prevent the complications.
\end{abstract}

Copy Right, IJAR, 2017,. All rights reserved.

\section{Introduction:-}

The increased tendency for thrombosis due to physiological changes in pregnancy is a recognized risk factor for deep vein thrombosis, stroke and pulmonary embolism during the antenatal period as well as in puerperium (Tatlisumak $\mathrm{T}$,2016).One such complication is cerebral venous thrombosis, presenting with severe headache, neurological deficits and seizures. The prognosis is good when diagnosis and management is not delayed.

For the obstetrician, it would also be important to rule out other causes of convulsions and headache in pregnancy (Tatlisumak T,2016). Clinical entities like thrombophilia, which can affect future pregnancies and can have an impact on the contraceptive choices, need to be identified in such cases. 
Cerebral venous thrombosis (CVT) is any thrombosis occurring in intracranial veins and sinus, which is a rare disorder (Tatlisumak T, 2016). It is more common in women than in men with a ratio of approximately 3:1 (Diana A de souse,2016). The prevalence of CVT in Indian population is about 4.5/ 1000 obstetric admissions (Prakesh C,2012).

However, the literature review shows that the risk of stroke and cerebrovascular complications are increased in pregnancy and puerperium compared to the non-pregnant women. Although pregnancy-associated stroke or CVT is uncommon, the risk of stroke is greatly increased above the low baseline rate in young patients during late pregnancy and, even more so, during the puerperium (Aniket S, 2011).

Haemorrhagic infarction can occur in the acute stage of CVT. The hypercoagulable state of pregnancy and puerperium are an important factors contributing to the risk of cerebral infracts and several risk factors have been implicated, which can predispose a pregnant women to cause cerebrovascular complications. CVT is an uncommon serious neurologic disorder in young gravidas requiring proper assessment of the potential differential diagnoses and prompt management (Sheeba M,2016).

\section{Objective:-}

To identify and evaluate the clinical features of pregnancy-related CVT populations, including disease onset and development, imaging features, diagnosis, treatments, and patient prognoses.

\section{Methods and Materials:-}

It is a Retrospective analytical study. The hospital records of all the women who are pregnant or in puerperium period with diagnosis of cerebrovascular thrombosis as revealed by MRI and were treated in R.L. Jalappa Hospital and research centre, Tamaka, Kolar were analysed retrospectively. The MRI was done on Seimen 1.5 T Scanner. The duration of the study was one year from October 2015 to October 2016. In these patients the clinical data, risk factors, neurological features, investigations, results and neuroimaging reports were analysed.

All the women who are pregnant or in puerperium period with the diagnosis of cerebrovascular thrombosis as revealed by MRI and were treated in R.L. Jalapa Hospital, Tamaka, Kolar was included in the study. Patients with previous history of cerebral venous thrombosis before pregnancy, and medical illness like hypertension, diabetic mellitus, Patients with history of vascular heart disease, hypercoagulable disease were excluded from the study.

\section{Results:-}

The mean age of our patients was 27 years old, ranging from 20 years to 35 years old. It occurred more in puerperium period than compared to pregnancy.

\section{Clinical presentation:-}

The mode of onset of symptoms was subacute in $32 \%$ of cases, acute in $48 \%$ of cases. The clinical presentation was variable, but the most common symptoms were headaches in all 6 cases (100\%), seizures in 6 cases (100\%) including 1 cases of status epilepticus, loss of consciousness was noticed in 1 case $(16.6 \%)$ and fever in 3 cases $(49.8 \%)$, motor disability in 1 cases $(16.6 \%)$.

\section{Biological and Radiological Examinations:-}

The biological examination revealed anemia in 4 cases $(66.4 \%)$, hyperleukocytosis in 3 cases (49.8\%), raised erythrocyte sedimentation rate in 3 cases $(49.8 \%)$. The CT-scan showed direct signs such as sagittal sinus thrombosis in only 1 cases $(16.6 \%)$. CT scan was found to be normal in 3 cases. All the patients were benefited to MRI examinations.

The MRI associated with MRV was more efficient in demonstrating the lesion compared to CT in all the cases. MRI shows wedge shaped lesion in the left occipital region in $1^{\text {st }}$ case, acute lacunar infract of anteromedial aspect of right thalamus left thalamocapsular region posterolateral aspect of left thalamus thrombosis of anterior $1 / 3$ of sagittal sinus in $2^{\text {nd }}$ case, sagittal sinus thrombosis in both case $3^{\text {rd }}$ and $4^{\text {th }}$ case, inferior sinus thrombosis in $5^{\text {th }}$ case and B/L thalami ( $\mathrm{L}>\mathrm{R})$ splenium of corpus callosum in the last case. 


\section{Etiology:-}

All patients underwent investigation of the thromboembolic antecedent, clinical examination, blood cells enumeration, prothrombin time (PT), activated partial thromboplastin time (APTT), erythrocyte sedimentation rate (ESR) and a thoracic x-ray systematically. The post-partum was the most frequent cause of CVT in our case series consisting of 4 cases (66.4\%); infectious origin was detected in 3 cases $(49.8 \%)$ which was revealed by raised in ESR and hyperluekocytosis ; anemia was found in 3 cases ( $49.8 \%$ ). No cases of preeclampsia, multiple gestation, $\mathrm{h} / \mathrm{o}$ any contraceptive used or any smoking history were seen among the patients.

\section{The treatment:-}

The treatment of intracranial hypertension were performed by carbonic anhydrase inhibitor or mannitol (83.2\%), and the lumbar puncture was done to remove the cerebrospinal fluid (16.6\%) to relieve the pressure symptoms. All of our patients (100\%) received heparin therapy (low-molecular-weight heparin 100\%) followed by oral anticoagulant. Antibiotics was administrated to patients with infectious origin (49.8\%). Antiepileptic (100\%) were treated for the cases of seizures. We used aspirin in 1 case (16.6\%). There was no maternal mortality in our study. In our study, surgery was not the treatment of choice in any of the reported cases.

\section{The outcome:-}

After treatment, a full recovery was noticed in 6 cases $(100 \%)$. There was no maternal mortality in the present study.

\section{Discussion:-}

Cerebral venous thrombosis is a rare type of stroke. It might occur at any age. The diagnosis of CVT requires a visualization of the thrombosis, which can be commonly demonstrated by cerebral MRI. The CT-scan might be helpful but does not allow its diagnosis.

CVT affects about five people per million per year and represents $0.5 \%$ of all stroke (Marie-Germaine Bousser , 2007). CVT might occur in any stage of life. Most authors agree that there is increase in incidence of CVT in pregnancy and in puerperium. Other previous studies have reported several predisposing risk factors for CVT including pregnancy, use of oral contraceptives, autoimmune disease, thrombotic factors, trauma, malignancy, and infections (Black.C, 2002;Niksirat A,2014; Dentali F,2006). Several studies revealed that taking oral contraceptive is associated with numerous major side effects including cerebrovascular, cardiovascular, and peripheral vascular disorders (Scoditti U, 1998;.Siritho S,2003;Jick H,1995). The role of OCP consumption has greatly considered and limited evidences reported an upper incidence of CVT among OCP users in Ramadan (Saidee M, 2008; . Sasannejad P,2012; Saadatnia M,2009). Headaches represent the main and first symptom of CVT retrieved in most studies including our study. Other symptoms include partial and generalized seizures, motor or sensitive deficits, altered consciousness, and papilledema which might be associated to other symptoms. The infarct is frequently hemorrhagic (10 to $50 \%$ of cases in the literature) [Crassard I,2003].

Most of the routine blood assessment does not have diagnostic value for CVT. However, it might help to establish its etiology. Several studies confirmed the elevation of the D-dimers during the CVT but their predictive negative value seems only interesting for patients having signs of encephalic reach. Therefore, an absence of D-dimers elevation in case of isolated headache cannot rule out CVT (Crassard Isabelle, 2005).

CVT is often multifactorial origin since several disorders might initiate or dispose patients. In the International studies of the Cerebral Vein and Dural Sinus Thrombosis (ISCVT), 44\% of the patients had shown more than one origin and predisposing factor including the congenital and genetic thrombophilia which was recorded in $22 \%$ patients (Ferro José M,2004). In developed countries, non-infectious origins such oral contraceptives, cancer, and thrombophilia are the most frequent causes with a prevalence of 80\%( Einhäupl KM, 1990; Biousse V, 1999)

The etiologies in our study was similar to the developing countries finding (Zhang Zaiqiang, 2000; DaifAbdulkader, 1995). Infections and post-partum causes constitute the most frequent causes. Several factors could be associated to the postpartum CVT, which can be caesarean section, arterial hypertension, and anemia. On the other hand, eclampsia is not a risk factor and no case of eclampsia has been counted in our study.

Heparin (low-molecular-weight heparin) is the first-line treatment even in presence of hemorrhagic infarction. In our study, $100 \%$ of patients benefited from anticoagulant treatment. Systemic or local thrombolysis may be used in CVT when patients deteriorate despite adequate anticoagulation and other causes of deterioration have been ruled out, thrombolysis or thrombectomy may be considered in specialized centers (Ciccone Alfonso, 2004; Wasay M, 2001). 
In our patients who consulted with raised intracranial pressure we used carbonic anhydrase inhibitor or mannitol. Antiepileptic and antibiotics are useful to treat seizures and infection.

\section{Conclusion:-}

CVT in pregnancy and in puerperium period is not uncommon. MRI is the key diagnostic tool. The outcome is favorable using heparin treatment. Timely recognition and early treatment can provide better prognosis and can help decrease maternal morbidity and maternal mortality.

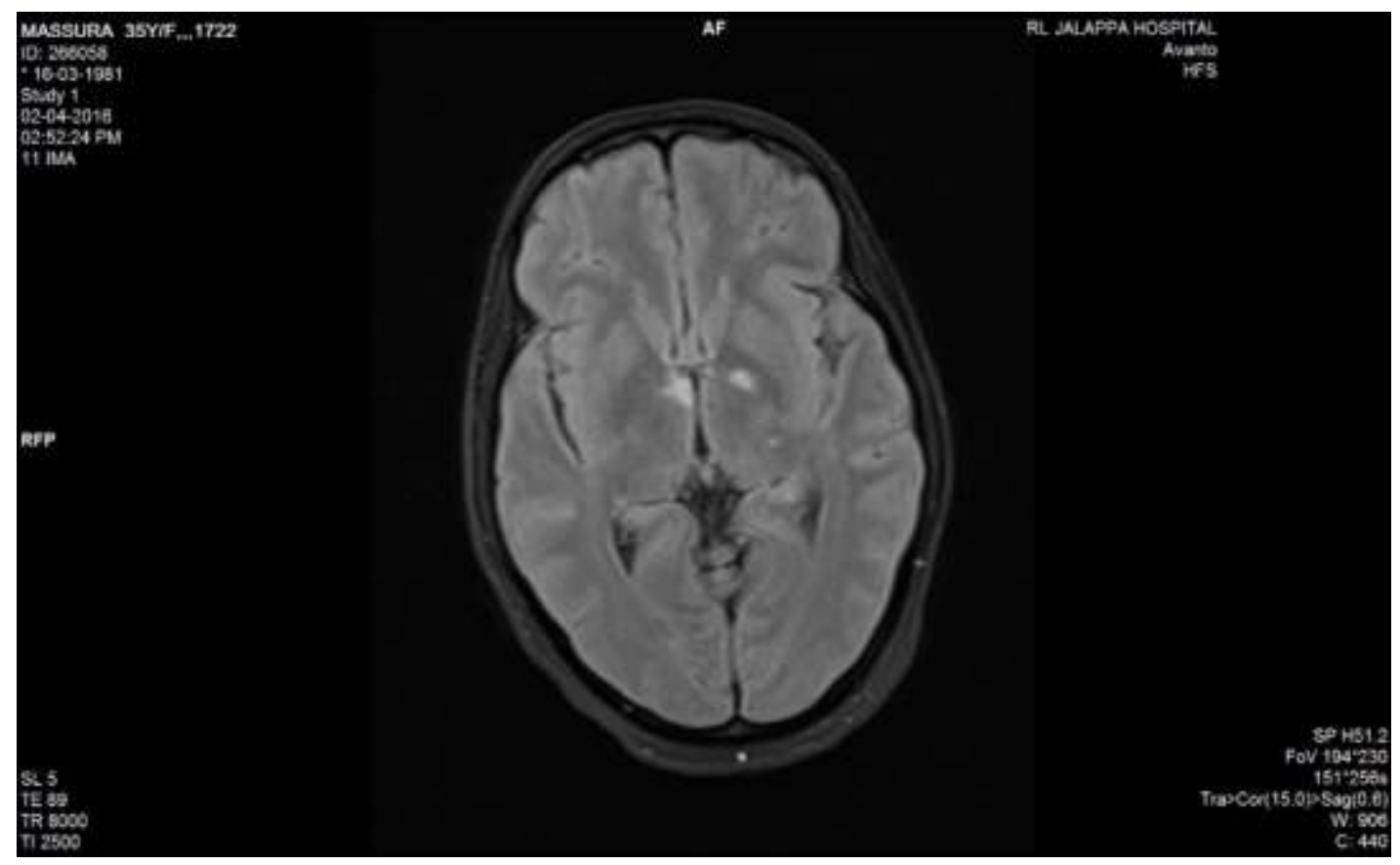

Fig.1 :- Axial flair showing acute lacunar infract of anteromedial aspect of right thalamus left thalamocapsular region postolateral aspect of left thlamus thrombosis.

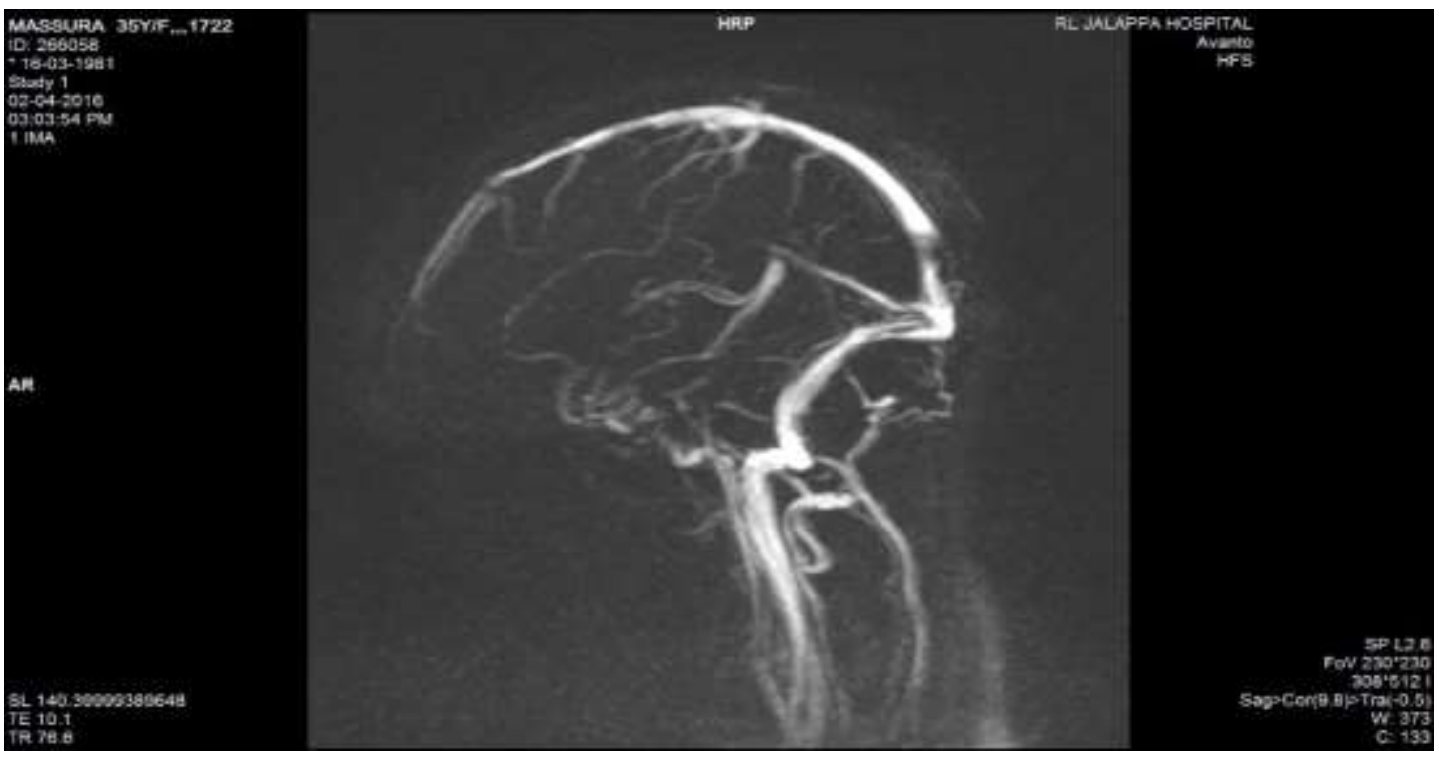

Fig. 2 :-Sagital view showing thrombosis of anterior $1 / 3$ of sagital sinus. 


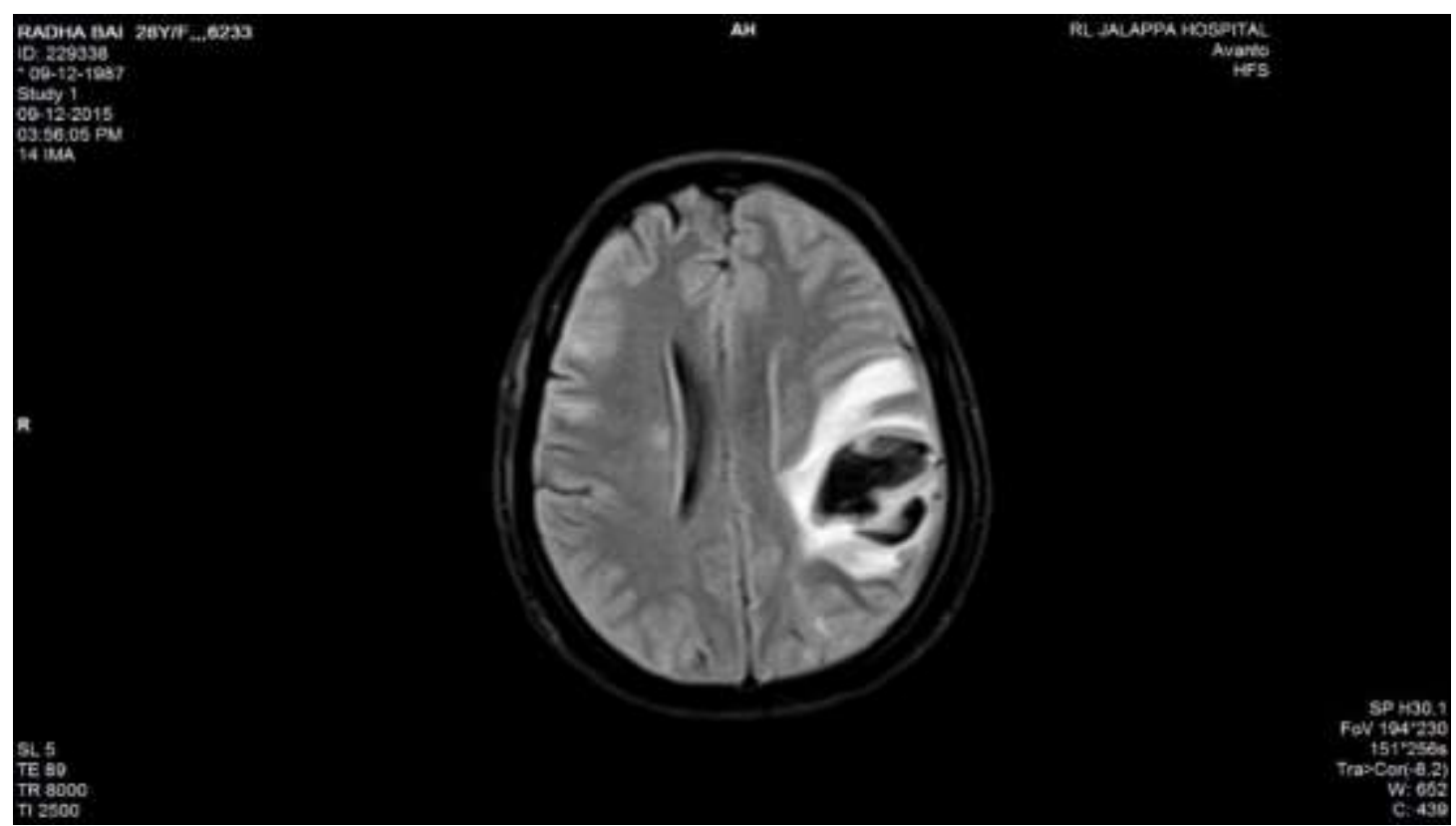

Fig.3 :- Axial view showing haemorrhagic lesion in left fronto-parietal and left occipital lobe

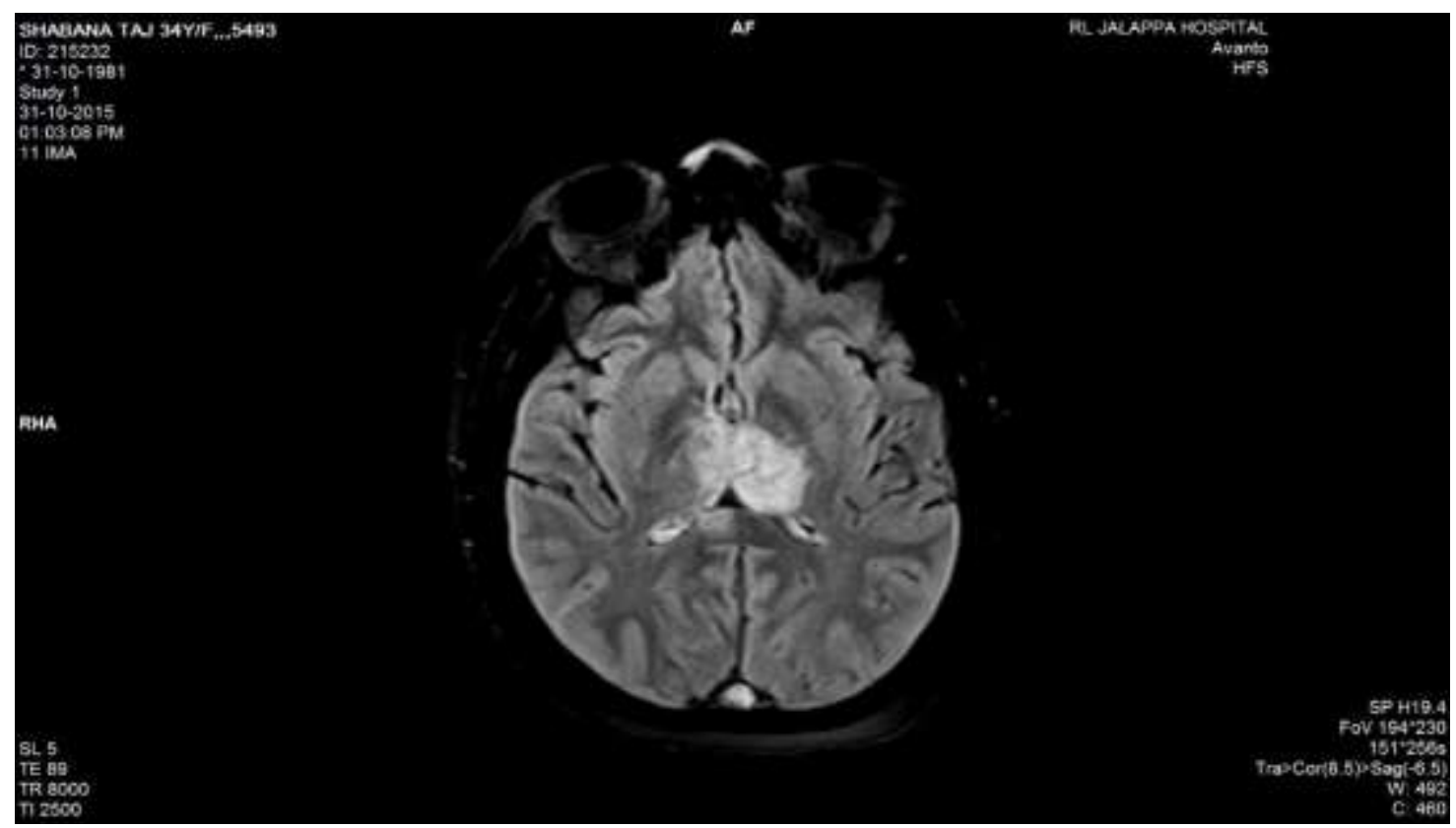

Fig 4 :- A xial view showing haemorrhagic infracts involving bilateral thalami (left> right) and splenium of corpus callosum with dural venous sinus thrombosis.

\section{References:-}

1. Aniket S. Kakade, Yashwant S. kulkarni. Cerebral venous thrombosis in pregnancy.BHJ. 2011; 53: 414-416.

2. Biousse V, Bousser MG. Cerebral venous thrombosis. Neurologist.1999;5:236-249.

3. Black.C, James.A ,Jick H. Clinical risk factors for venous thromboembolus in users of the combined oral contraceptive pill. Br J ClinPharmacol 2002; 53(6): 637-40.

4. Crassard I, Ameri A, Rougemont D, Bousser MG. Thrombosesveineusescérébrales. EncyclMédChirNeurologie. 2003; 17- 046-R-10 : 12.

5. Crassard Isabelle, Soria Claudine, Tzourio Christophe, Woimant France, et al. A Negative D-Dimer Assay Does Not Rule Out Cerebral Venous Thrombosis. A Series of Seventy-Three Patients. Stroke.2005;36:1716 
6. Ciccone Alfonso, Canha O Patricia, Falca O Filipa, Ferro Jose' M, Sterzi Roberto. Thrombolysis for Cerebral Vein and Dural Sinus Thrombosis. Stroke.2004;35:2428.

7. DaifAbdulkader. Cerebral Venous Thrombosis in Adults: A Study of 40 Cases from Saud Arabia. Stroke. 1995;26(7):1193-1195.

8. Diana A de souse, Patricia C, Jose M. Safety of pregnancy after Cerebral Venous Thrombosis.AHA.2016;47:713-718.

9. Dentali F, Gianni M, Crowther MA, Ageno W. Natural history of cerebral vein thrombosis: a systematic review. Blood 2006; 108(4): 1129-34.

10. Einhäupl KM, Villringer A, Habert RL. Clinical spectrum of sinus venous thrombosis in Cerebral sinus thrombosis: experimental and clinical aspects. 1990.

11. Ferro José M, CanhãoPatrícia, Stam Jan, Bousser Marie- Germaine, Barinagarrementeria Fernando. Prognosis of Cerebral Vein and Dural Sinus Thrombosis Results of the International Study on Cerebral Vein and Dural Sinus Thrombosis (ISCVT). Stroke. 2004;35(3):664-670.

12. Jick H, Jick SS, Gurewich V, Myers MW, Vasilakis C. Risk of idiopathic cardiovascular death and nonfatal venous thromboembolism in women using oral contraceptives with differing progestagen components. Lancet 1995; 346(8990): 1589-93.

13. Marie-Germaine Bousser, José M Ferro. cerebral venous thrombosis: an update. Lancet Neurol. 2007; 6(2): 162-70.

14. Niksirat A, Ghoreishy A, ShoghliAR,Kolifarhood G, Yousefian F. Incidence ofcerebral venous thrombosis in Iranian women: a longitudinal two-year study in Zanjan province of Iran. J Pioneer Med Sci2014; 4(1): $28-31$.

15. Prakesh C, Bansal BC. Cerebral Venous Thrombosis. JIACM: 2012;5(1). 55-61.

16. Saadatnia M, Zare M, Fatehi F, AhmadiA.The effect of fasting on cerebral venous and Dural sinus thrombosis. Neurol Res 2009;31(8): 794-8.

17. Saidee M, Froghipoor M, Sasannejad P, MellatArdakani A, Azarpazhooh MR. The relation between short course oral contraceptive consumption and cerebral vein thrombosis in Ramadan. Iran J Neurol 2008; 7(23): 260-5.

18. Sasannejad P, Mellat AA, Velayati A, Shoeibi A, Saeidi M, Foroughipour M, et al. Cerebral vein thrombosis in women using short course oral contraceptive consumption. Iran J Reprod Med 2012;10(6): 537-42.

19. Scoditti U, Buccino GP, Pini M, Pattacini C, Mancia D. Risk of acute cerebrovascular events related to low oestrogen oral contraceptives treatment. Ital J NeurolSci 1998; 19(1): 15-9.

20. Sheeba M, Harsha S, Sumedha G, Manjula S, Pratime M. Cerebral Venous Thrombosis in Pregnancy - A Poignant Allegory of an unsual case. JCDR. 2016; 10 (12): 8-9.

21. Siritho S, Thrift AG, McNeil JJ, You RX,Davis SM, Donnan GA. Risk of ischemic stroke among users of the oral contraceptive pill: The Melbourne Risk Factor Study (MERFS) group. Stroke 2003; 34(7): 1575-80.

22. Prakesh C, Bansal BC. Cerebral Venous Thrombosis. JIACM: 2012;5(1). 55-61.

23. Tatlisumak T, Jood K, Putaala J. Cerebral Venous Thrombosis Epidemiology in Change. AHA. 2016; 47: 21692170.

24. Wasay M, Bakshi R, Kojan S, Bobustuc G, Dubey N, Unwin DH. Nonrandomized Comparison of Local Urokinase Thrombolysis Versus Systemic Heparin Anticoagulation for Superior Sagittal Sinus Thrombosis. Stroke. 2001;32(10):2310-2317.

25. Zhang Zaiqiang et al. Cerebral venous sinus thrombosis: a clinical study of 23 cases. Chinese Medical journal. 2000. 113 (11); 1043-1045. 